\title{
Correlation Analysis between Ocular Surface Parameters with Subjective Symptom Severity in Dry Eye Disease
}

\author{
Jee Hye Lee, Chang Hwan Kim, Chul Myong Choe, Tae Hoon Choi \\ Nune Eye Hospital, Seoul, Korea
}

Purpose: To evaluate the clinical symptoms of patients with dry eyes, based on the ocular surface disease index (OSDI) and analyze the relationship between OSDI and various ocular surface parameters.

Methods: This was a retrospective study that included 45 eyes of 45 dry eye patients who visited the Seoul Nune Eye Hospital from August 2017 to December 2017. The patients were assessed by non-invasive keratography for the first break-up time, lipid layer thickness (LLT), tear osmolarity, tear matrix metalloproteinase-9 immunoassay as well as with the conventional Schirmer I test and fluorescein break-up time. The patient's symptoms were evaluated by the OSDI questionnaires and correlations were analyzed based on the parameters described above.

Results: There were significant negative correlations between OSDI and non-invasive keratography for the first break-up time ( $p=0.038, r=-0.330)$, and LLT ( $p=0.005, r=-0.426)$. However, there were no significant correlations between OSDI and fluorescein break-up time, Schirmer I score, and tear osmolarity $(p=0.173,0.575$, and 0.844 respectively). OSDI was not significantly different between matrix metalloproteinase- 9 positive and negative groups $(p=0.768)$.

Conclusions: Non-invasive examinations such as non-invasive keratograph break-up time and interferometry of LLT can be efficient tools for evaluating dry eye symptoms.

Key Words: Diagnosis, Dry eye syndromes, Tears

Dry eye is a multifactorial disease related to impaired tear film homeostasis and is accompanied by tear film instability and hyperosmolarity, and ocular surface inflammation [1]. One characteristic feature of dry eye disease is the discordance between the patient's symptoms and clinical signs, which makes dry eye disease treatment more challenging [2]. As part of continuing efforts to minimize this gap between symptoms and signs, various emerging

Received: November 12, 2019 Final revision: December 16, 2019

Accepted: January 3, 2020

Corresponding Author: Tae Hoon Choi, MD, PhD. Nune Eye Hospital, 404 Seonreung-ro, Gangnam-gu, Seoul 06198, Korea. Tel: 82-1661-1175, Fax: 82-2-2086-7779, E-mail: thchoi@hanmail.net technologies have been developed such as a tear matrix metalloproteinase-9 (MMP-9) immunoassay, the tear osmolarity test, and some diagnostic imaging methods, including corneal topography, ocular coherence tomography, meibography, and interferometry, as well as conventional tear break-up time (TBUT), the Schirmer test, and an ocular surface staining score. Moreover, these testing methods have become more accessible and are already widely used.

Several previous studies evaluated the correlation between the ocular surface disease index (OSDI) and objectively quantifiable parameters in dry eye patients $[3,4]$. They examined dry eye parameters including conventional fluorescein TBUT (FBUT), the Schirmer test, cornea and 
conjunctival staining score, blepharitis severity grade, and/ or lipid layer thickness (LLT) by Lipiview (TearScience, Morrisville, NC, USA), to evaluate correlations between results and OSDI. They concluded that meibomian gland dysfunction should be an important factor that influences subjective pain and discomfort in dry eye patients. However, to our knowledge, no studies have evaluated additional further ocular surface parameters, including the tear MMP-9 immunoassay using InflammaDry (RPS Diagnostics, Sarasota, FL, USA), tear osmolarity using the TearLAB Osmolarity system (TearLab Corporation, San Diego, CA, USA), and the non-invasive keratograph TBUT using Keratograph 5M (Oculus, Wetzler, Germany), in addition to the parameters outlined above. We examined almost all ocular surface parameters using appropriate techniques to analyze the correlation between OSDI and these parameters.

\section{Materials and Methods}

A chart review of the 45 patients who presented at the Seoul Nune Eye Hospital between August 2017 to December 2017 due to dryness in their eyes and were diagnosed with dry eye disease was performed. The diagnosis of dry eye disease was made when the patients experienced dry eye symptoms and the FBUT was shorter than 10 seconds and/or cornea or conjunctival superficial erosion was seen in slit-lamp examination by the same examiner (THC). We excluded patients with conditions that could affect dry eye parameters such as contact lens use, history of refractive, glaucoma, or retina surgery, cataract surgery within 6 months, systemic disease including diabetes, autoimmune disease, cranial nervous system disorders, and use of ocular medications other than artificial tears. Approval from the institutional review board of Nune Eye Hospital was obtained (N-1802-002-999) and informed consent was waived because of the retrospective nature of the study.

Forty-five subjects were examined with non-invasive keratograph first break-up time (NIKf-BUT), LLT, tear osmolarity, tear MMP-9 immunoassay, FBUT, and Schirmer I score assessments. After several blinks, the NIKf-BUT, which is determined as the time between the blink and the first mire distortion, was measured by Keratograph 5M. A Lipiview provides data for the minimum, maximum, average LLT, and partial blink rate. The average LLT was used
Table 1. Demographics and ocular surface parameters of the study subjects with dry eye disease

\begin{tabular}{lc}
\hline Characteristics & Value \\
\hline Eyes & 45 \\
Male : female & $9: 36$ \\
Age (yr) & $51.5 \pm 14.4$ \\
Schirmer I score (mm) & $12.7 \pm 9.1$ \\
FBUT (sec) & $5.2 \pm 2.3$ \\
NIKf-BUT (sec) & $5.8 \pm 2.0$ \\
LLT (nm) & $77.8 \pm 17.8$ \\
Tear osmolarity (mOsm/L) & $303.9 \pm 16.9$ \\
Tear MMP-9 positive : negative & $17: 22^{*}$ \\
OSDI & $38.3 \pm 20.1$ \\
\hline
\end{tabular}

Values are presented as number or mean \pm standard deviation. FBUT = fluorescein break-up time; NIKf-BUT = non-invasive keratograph first break-up time; LLT = lipid layer thickness; MMP-9 = matrix metalloproteinase-9; OSDI = ocular surface disease index.

"Due to missing data.

in this study because the average LLT provided a more appropriate representation of tear film stability than minimum or maximum LLT [5]. A tear sample from the patient's palpebral conjunctiva was collected and the osmolarity was measured with the TearLAB Osmolarity system. An InflammaDry tear immunoassay test was performed to detect tear MMP-9. A sampling fleece was used to collect tears by gently dabbing multiple locations of palpebral conjunctiva, then a sample collector was assembled onto the test cassette and the test was interpreted after 10 minutes had elapsed. The presence of one blue line and one red line, regardless of the intensity of the red line in the test's result window, was regarded as a positive test result. The FBUT was measured as the time interval between fluorescein staining and the appearance of the first dry spot using the cobalt blue filter. A Schirmer I test was performed without topical anesthesia by placing the standardized strip in the lower lid temporal side for 5 minutes. Thirty-minute intervals between all measurements ensured subsidence of effects for each examination. Only the right eye was studied in this current study. OSDI (Allergan, Irvine, CA, USA) questionnaires were used to assess patient dry-eye symptoms.

Continuous variables were expressed as mean \pm standard deviation. Pearson correlation analysis was used to evalu- 
A

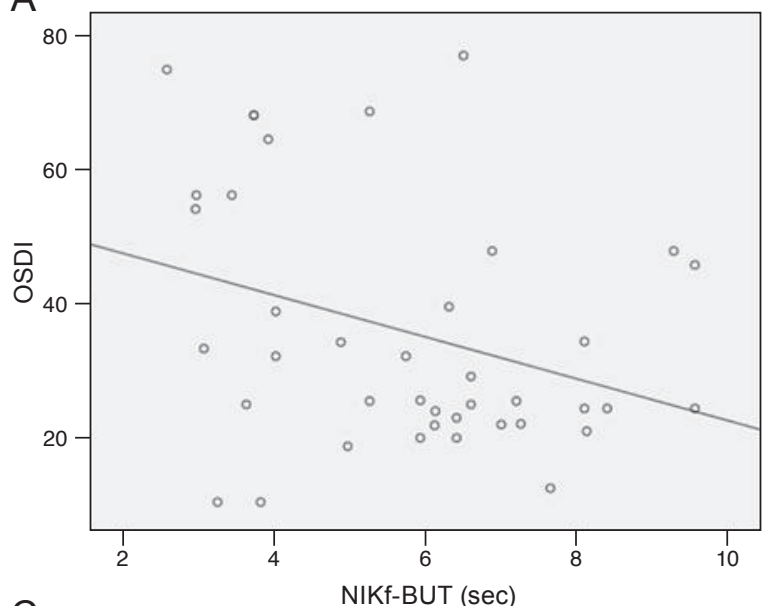

C

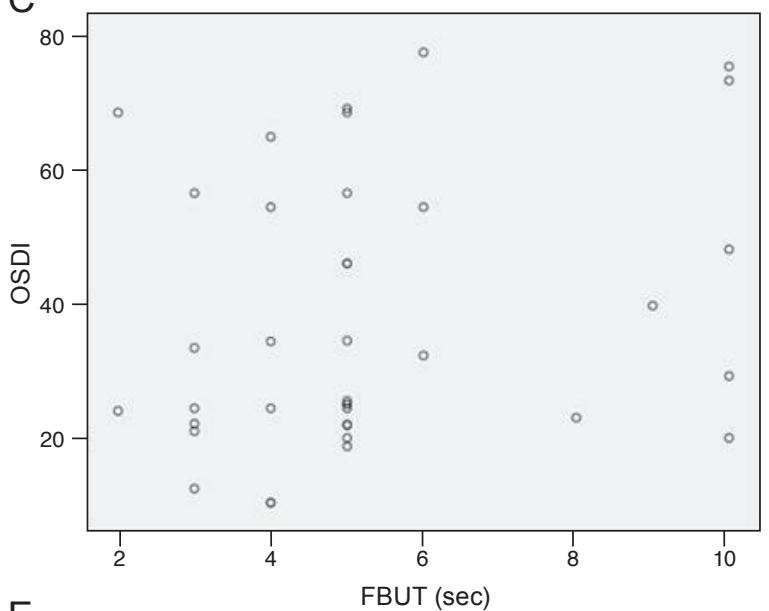

E

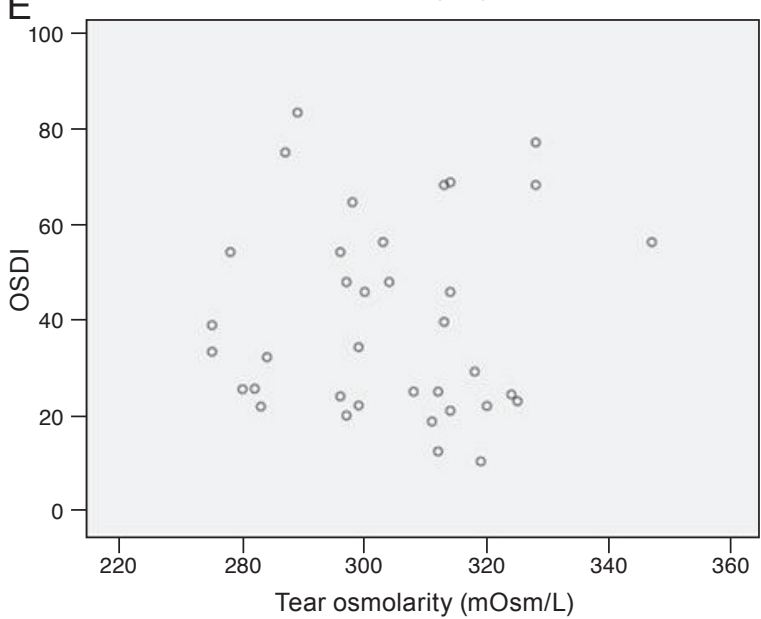

$\mathrm{B}$

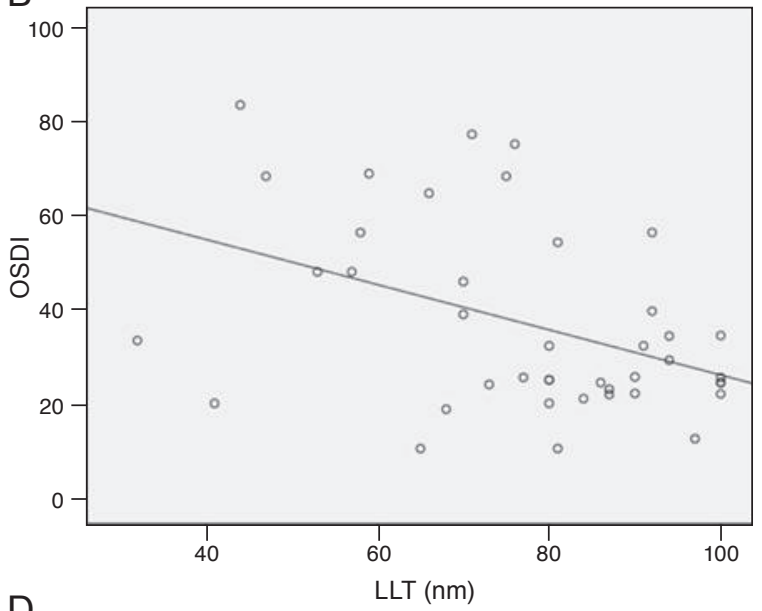

$\mathrm{D}$

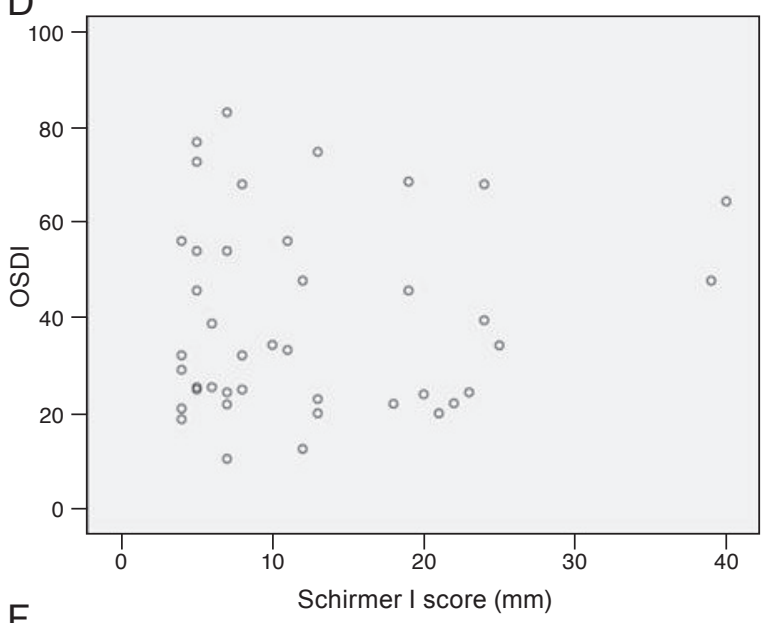

$\mathrm{F}$

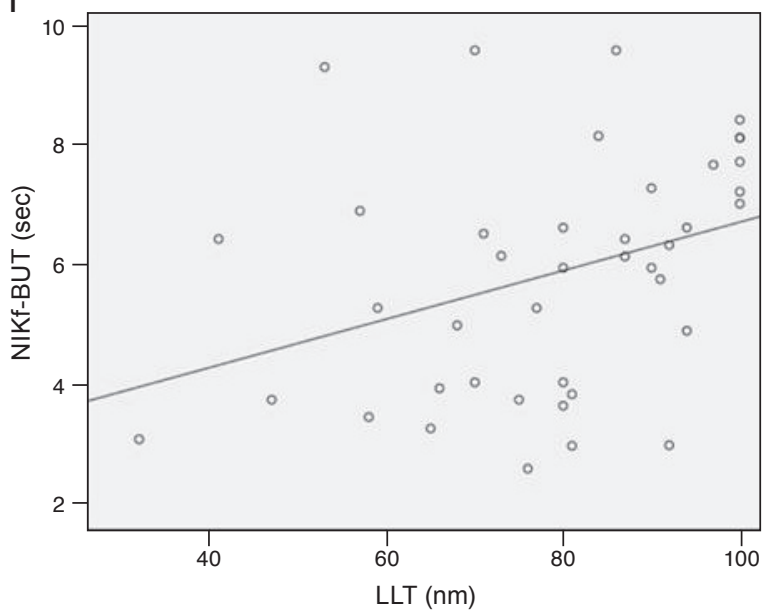

Fig. 1. Pearson correlation scatter plot for (A) non-invasive keratograph first break-up time (NIKf-BUT) and ocular surface disease index (OSDI), (B) lipid layer thickness (LLT) and OSDI, (C) fluorescein break-up time (FBUT) and OSDI, (D) Schirmer I score and OSDI, (E) tear osmolarity and OSDI, (F) LLT and NIKf-BUT. (A), (B), and (F) showed significant correlations: $(\mathrm{A}) r=-0.330, p=0.038$; $(\mathrm{B}) r=$ $-0.426, p=0.005 ;(\mathrm{F}) r=0.353, p=0.024$.

ate correlation between the OSDI and ocular surface parameters. An independent $t$-test was used to compare the
OSDI between the MMP-9 positive group and negative group. In all statistical tests, a $p$-value $<0.05$ was consid- 
Table 2. Correlation analysis between the ocular surface disease index and ocular surface parameters

\begin{tabular}{|c|c|c|c|c|c|}
\hline & FBUT & Schirmer I score & NIKf-BUT & LLT & Tear osmolarity \\
\hline Pearson correlation coefficient & 0.226 & 0.090 & -0.330 & -0.426 & 0.034 \\
\hline$p$-value & 0.173 & 0.575 & $0.038^{*}$ & $0.005^{*}$ & 0.844 \\
\hline
\end{tabular}

FBUT $=$ fluorescein break-up time; NIKf-BUT = non-invasive keratograph first break-up time; LLT = lipid layer thickness. ${ }^{*} p<0.05$.

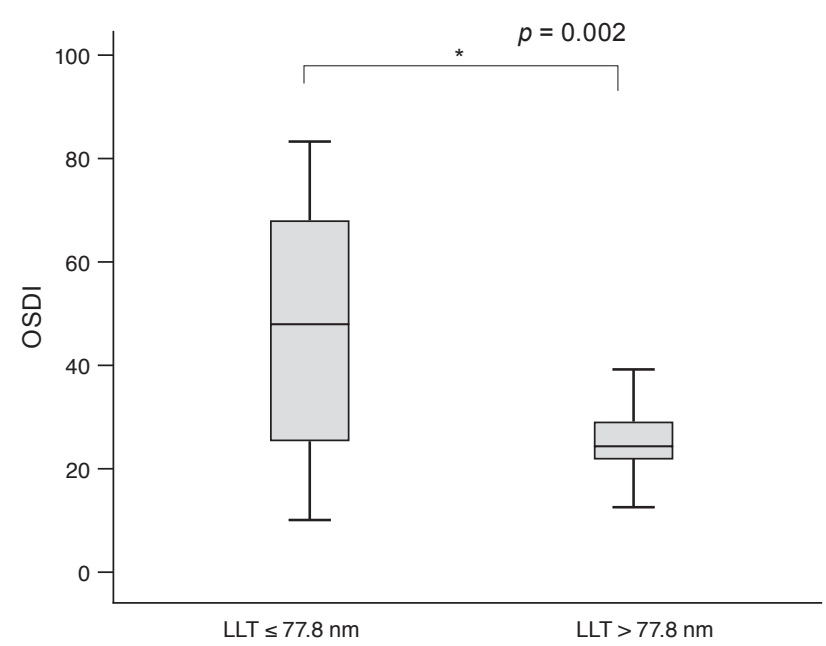

Fig. 2. Comparison of the ocular surface disease index (OSDI) between the above-average lipid layer thickness (LLT) group and below-average LLT group. The mean average LLT was $77.8 \pm 17.8$ $\mathrm{nm}$. A $p$-value was calculated by independent $t$-test.

ered statistically significant. Data was analyzed using PASW Statistics ver. 18 (SPSS Inc., Chicago, IL, USA).

\section{Results}

The mean age of the patients was $51.5 \pm 14.4$ years and 36 of 45 patients were female. The mean FBUT was $5.2 \pm$ 2.3 seconds, the mean Schirmer I score was $12.7 \pm 9.1 \mathrm{~mm}$, and the mean OSDI was $38.3 \pm 20.1$. The demographic characteristics and dry eye parameters are summarized in Table 1. All patients were considered level 1 or 2 based on the Korean Corneal Disease Study Group guidelines for the diagnosis of dry eye [6].

Among parameters that included the FBUT, Schirmer I score, NIKf-BUT, LLT, tear osmolarity, and OSDI, OSDI showed significant negative correlations with NIKf-BUT $(p=0.038, r=-0.330)$ and LLT $(p=0.005, r=-0.426)(\mathrm{Ta}-$ ble 2 and Fig. 1A, 1B). Other dry eye parameters such as

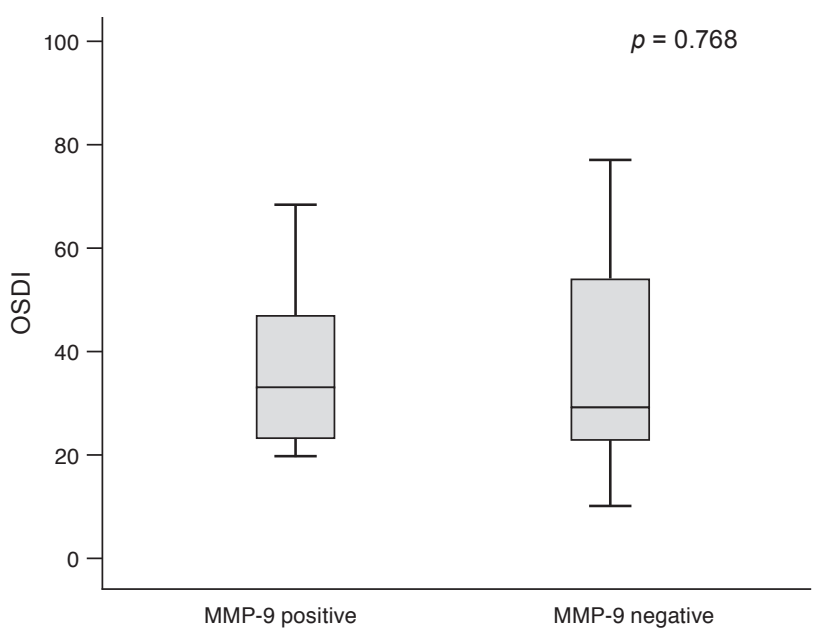

Fig. 3. Comparison of the ocular surface disease index (OSDI) between the tear matrix metalloproteinase-9 (MMP-9) immunoassay positive group and negative group. A $p$-value was calculated by independent $t$-test.

FBUT, Schirmer I score, and tear osmolarity did not show significant correlations with OSDI ( $p=0.173,0.575,0.844$, respectively) (Table 2 and Fig. 1C-1E). There was also a positive correlation between NIKf-BUT and LLT ( $p=$ $0.024, r=0.353$ ) (Fig. 1F). The OSDI was significantly higher in the below-average LLT group (LLT $\leq 77.8$, mean LLT was $77.8 \pm 17.8 \mathrm{~nm}$ ) than in the above-average group (LLT >77.8) $(p=0.002)$ (Fig. 2). The mean OSDI was 40.1 \pm 19.0 in the MMP-9 positive group and $38.2 \pm 20.9$ in the MMP-9 negative group. There was also no significant difference in the OSDI of the MMP-9 positive group and MMP-9 negative group ( $p=0.768$ ) (Fig. 3).

\section{Discussion}

Impaired tear homeostasis, which is related to the amount of tears produced, the rate of tear evaporation, goblet cell density, and the presence or absence of inflam- 
mation, can lead to dry eye [7]. Dry eye diseases have typically been evaluated by patient's subjective symptoms, tear production, tear film instability, and damaged cornea and conjunctival status [6]. Previous studies have investigated parameters that could be associated with subjective discomfort of dry eye [3,4]. In contrast with expectations, the studies revealed that TBUT, which is related to tear film instability, was not significantly correlated with OSDI. The TBUT was measured by fluorescein dye instillation in their studies and in this study, and the FBUT was not associated with OSDI. However, NIKf-BUT was significantly correlated with OSDI in this study ( $p=0.038, r=-0.330$ ). This is because a non-invasive examination would exclude fluorescein's interference in tear stability and could be measured with more consistency. Moreover, non-invasive keratograph break-up time (NIKBUT) was associated with greater repeatability in dry eyes than in non-dry eyes because non-dry eyes need to be opened longer during examination due to longer TBUT which induces more reflex tear secretion [8].

FBUT is a widely-used conventional diagnostic method for tear film instability. However, there are some limitations to FBUT because fluorescein can destabilize the tear film and measurements can be affected by examiners [9]. The Keratograph 5M can measure NIKBUT by recording a real time image of the entire tear break-up course and measure the time between the blink and the mire distortion. Twenty-two placido rings are reflected to the cornea and their distortions are analyzed within 192 divided sections and visualized by a color-coded map. The timing of the first break in the tear film, NIKf-BUT and the average time of all the break-ups during the measurement (non-invasive keratograph average break-up time) are displayed. NIKf-BUT was used in this study and the mean NIKfBUT was $5.8 \pm 2.0$ seconds, which was longer than the FBUT, which was $5.2 \pm 2.3$ seconds. However, previous studies showed that the NIKBUT is shorter than the FBUT because software analysis of NIKBUT may detect very early tear film changes and heat from the LED (light emitting diode) may induce more tear break-ups $[8,10,11]$. In this study, the examiner subjectively measured the FBUT and did not use a stopwatch, which might have influenced these conflicting results.

LLT was also significantly correlated with the OSDI ( $p=$ $0.005, r=-0.426$ ). Blackie et al. [12] also revealed that LLT was significantly correlated with standard patient evalua- tion of eye dryness. However, Seo et al. [3] and Kim et al. [5] found that LLT was not correlated with OSDI. Whether the LLT is associated with OSDI remains controversial because different study designs or patient populations have reported conflicting results, however, the blepharitis severity score [4] or quality of the meibum [3] was significantly correlated with the OSDI. Meibomian gland dysfunction, one of the most important factors of dry eye, promotes tear film evaporation and decreases TBUT [13]. Several previous studies showed that TBUT and LLT are significantly correlated [5,14-16], and our study also confirmed that there was a significant association between NIKf-BUT and LLT ( $p=0.024, r=0.353$ ). Insufficient LLT associated with tear film instability might result in patient discomfort with a high OSDI.

Other ocular surface parameters such as the Schirmer I score, tear osmolarity, and tear MMP-9 presence were not associated with OSDI. Because this study excluded autoimmune disease patients and the mean Schirmer I score was relatively high at $12.7 \pm 9.1 \mathrm{~mm}$, the Schirmer I score might not show significant correlations with OSDI. MMP9 is an ideal inflammation marker because it is induced by all of the primary mediators, including IL-1 13 , TNF- $\alpha$, IL6, IL-8, IL-17 and accumulates throughout the inflammatory cascade [17-19]. MMP-9 destabilizes tear film, contributes to corneal barrier dysfunction which causes epithelial cell desquamation, and results in pain and fluctuating vision [19]. However, the results of our study and other studies have indicated that there is no significant difference in the symptoms and signs of dry eye patients between MMP-9 positive and negative groups [20]. Hyperosmolarity of the tear film has been proposed as one of the key pathogenic factors in dry eye disease, but a high variability of measurements and specific cut-off values to diagnose dry eye have not been established. Correlations between tear osmolarity and OSDI are currently controversial $[21,22]$.

There were several limitations to this study. Performing multiple non-invasive examinations such as NIKBUT and interferometry for LLT, as well as invasive examinations such as the Schirmer I test, FBUT, tear osmolarity, and MMP-9 immunoassay in one day could affect the test measurements, in spite of interval times between each examination. In addition, this study did not analyze the subjects based on dry eye severity because only relatively mild dry eye participants were enrolled. Gross examina- 
tions such as the cornea and the conjunctival staining score, blepharitis severity, or meibum quality were not evaluated as parameters. Due to small number of patients who showed cornea or conjunctival superficial erosions, results were not available for analyzing the association between OSDI and the ocular surface staining score. Despite these limitations, this study analyzed almost all ocular surface parameters that can be examined recently and determined whether OSDI was associated with NIKf-BUT or LLT, and also found that NIKf-BUT and LLT were significantly correlated with each other. The results of this study indicate that these non-invasive parameters could explain patient discomfort, which can influence clinicians to select appropriate clinical management strategies. Moreover, because these parameters can be visualized, patients can easily recognize their conditions and might feel validated when discussing concerns with their providers.

\section{Conflict of Interest}

No potential conflict of interest relevant to this article was reported.

\section{References}

1. Craig JP, Nichols KK, Akpek EK, et al. TFOS DEWS II definition and classification report. Ocul Surf 2017;15:27683.

2. Wilson SE, Stulting RD. Agreement of physician treatment practices with the international task force guidelines for diagnosis and treatment of dry eye disease. Cornea 2007;26:284-9.

3. Seo MH, Shin JY, Lee DH, Kim JH. Objective parameters associated with subjective symptom severity in dry eye syndrome patients. J Korean Ophthalmol Soc 2017;58:25967.

4. Moon IH, Kim TI, Seo KY, et al. The relationship between subjective ocular discomfort and blepharitis severity in dry eye patients. J Korean Ophthalmol Soc 2016;57:1507-13.

5. Kim RY, Na KS, Park YL, Kim HS. Correlation analysis of tear film lipid layer thickness and ocular surface disease index. J Korean Ophthalmol Soc 2017;58:788-96.

6. Hyon JY, Kim HM, Lee D, et al. Korean guidelines for the diagnosis and management of dry eye: development and validation of clinical efficacy. Korean J Ophthalmol 2014;28:197-206.

7. Sambursky R. Presence or absence of ocular surface inflammation directs clinical and therapeutic management of dry eye. Clin Ophthalmol 2016;10:2337-43.

8. Best N, Drury L, Wolffsohn JS. Clinical evaluation of the Oculus Keratograph. Cont Lens Anterior Eye 2012;35:171-4.

9. Mengher LS, Bron AJ, Tonge SR, Gilbert DJ. Effect of fluorescein instillation on the pre-corneal tear film stability. Curr Eye Res 1985;4:9-12.

10. Jiang Y, Ye H, Xu J, Lu Y. Noninvasive Keratograph assessment of tear film break-up time and location in patients with age-related cataracts and dry eye syndrome. $J$ Int Med Res 2014;42:494-502.

11. Jeong S, Lee SB. Reliability of a new non-invasive tear film break-up time measurement using a keratograph. J Korean Ophthalmol Soc 2016;57:1354-60.

12. Blackie CA, Solomon JD, Scaffidi RC, et al. The relationship between dry eye symptoms and lipid layer thickness. Cornea 2009;28:789-94.

13. Kim DW, Kwon YA, Song SW, et al. Clinical usefulness of a thermal-massaging system for treatment of dry eye with meibomian gland dysfunction. J Korean Ophthalmol Soc 2013;54:1321-6.

14. Nichols JJ, Nichols KK, Puent B, et al. Evaluation of tear film interference patterns and measures of tear break-up time. Optom Vis Sci 2002;79:363-9.

15. Isreb MA, Greiner JV, Korb DR, et al. Correlation of lipid layer thickness measurements with fluorescein tear film break-up time and Schirmer's test. Eye (Lond) 2003;17:7983.

16. Craig JP, Tomlinson A. Importance of the lipid layer in human tear film stability and evaporation. Optom Vis Sci 1997;74:8-13.

17. Na KS, Mok JW, Kim JY, et al. Correlations between tear cytokines, chemokines, and soluble receptors and clinical severity of dry eye disease. Invest Ophthalmol Vis Sci 2012;53:5443-50.

18. Massingale ML, Li X, Vallabhajosyula M, et al. Analysis of inflammatory cytokines in the tears of dry eye patients. Cornea 2009;28:1023-7.

19. Chotikavanich S, de Paiva CS, Li de Q, et al. Production and activity of matrix metalloproteinase- 9 on the ocular surface increase in dysfunctional tear syndrome. Invest Ophthalmol Vis Sci 2009;50:3203-9.

20. Lanza NL, McClellan AL, Batawi H, et al. Dry eye profiles 
in patients with a positive elevated surface matrix metalloproteinase 9 point-of-care test versus negative patients. Ocul Surf 2016;14:216-23.

21. Mathews PM, Karakus S, Agrawal D, et al. Tear osmolarity and correlation with ocular surface parameters in pa- tients with dry eye. Cornea 2017;36:1352-7.

22. Karakus S, Akpek EK, Agrawal D, Massof RW. Validation of an objective measure of dry eye severity. Transl Vis Sci Technol 2018;7:26. 